\title{
SENTINEL LYMPH NODE BIOPSY AS A PROGNOSTIC TOOL IN EARLY ORAL CANCER USING METHYLENE BLUE DYE
}

\author{
Mohamed M. Elshamaa ${ }^{1 *} M D$, Mohamed M. Fata ${ }^{2} P h D$, Gamal A. Swaify ${ }^{2} P h D$, Sherif \\ El-S. Hegab ${ }^{3} P h D$, Hanan Y. Tayel ${ }^{4} P h D$.
}

\begin{abstract} predicting subclinical neck nodal metastases in head and neck cancers. of occult neck micrometastasis and confirmation of the role of SLN in post-surgical follow up. negative lymph nodes. $\%)$ and NPV (100 \%) of histopathology at the loss of specificity (87.5 \%) and PPV (62.5 \%). complications for N0 patients by reducing the incidence or extent of neck dissection.

KEYWORDS: Sentinel L.N in H\& N, Early Oral Cancer, Prophylactic Neck Dissection.

RUNNING TITLE: Sentinel Lymph Node Biopsy in Early Oral Cancer.

1Assistant lecturer at Oral and Maxillofacial Surgery Department, Faculty of Dentistry, Beni Suef, University, Beni Suef, Egypt.

2Professor of Maxillofacial and Plastic Surgery Faculty of Dentistry, Alexandria University, Alexandria, Egypt.

3Professor of Radiodiagnosis and Interventional Radiology Faculty of Medicine, Alexandria University, Alexandria, Egypt.

4Professor of Pathology Department, Faculty of Medicine, Alexandria University, Alexandria, Egypt.
\end{abstract}

INTRODUCTION: Limited availability of lymphoscintigraphy facilities in Egypt and developing countries requires exploration of alternative methods of SLN detection. Sentinel Lymph Node (SLN) biopsy using a combination of radioisotopes and methylene blue dyes (MBD) have a good accuracy rate in

OBJECTIVES: Evaluation of the effectiveness of SLN using MBD alone and frozen section of early oral cancer with N0 neck patients in the identification

MATERIALS AND METHODS: 12 Patients with cN0 early (T1, T2) oral squamous cell cancers underwent SLN biopsy using peri tumoural MBD injection. Stained SLN nodes were sent for frozen section analyses. Patients had microscopic metastases in SLN underwent modified radical neck dissections and the rest underwent selective neck dissections. Paraffin sections were performed in all cases and immune-histochemistry (IHC) studies were performed on

RESULTS: Three cases (25\%) SLN couldn't be detected, while nine cases (75\%) the blue sentinel lymph node could be identified. Preoperative ultrasound examination of only two cases were not similar to the intraoperative frozen histopathology which reveals the presence of micrometastasis, therefore functional neck dissection was indicated in these two cases and all the frozen sections histopathology were similar to the postoperative paraffin sections. Considering HPE on paraffin fixed blocks as the gold standard, FS had a sensitivity, specificity, and NPV of $95.8 \%$. IHC with cytokeratin increased the sensitivity (100

CONCLUSION: SLN biopsy is useful and reliable for patients with early oral cancer that can benefit from reducing unnecessary functional and esthetic

* Corresponding Author:

Email: drmelshamaa@gmail.com

\section{INTRODUCTION}

Squamous cell carcinoma of the oral cavity represents about $2 \%$ of all malignant neoplasms and $47 \%$ of those developing in the head and neck area. The tongue is the most common site involved (1). Sentinel lymph node biopsy (SLNB) is based on the premise that malignancy proceeds from the primary tumor, to the sentinel lymph node (SLN), and then into the remaining regional lymphatics. (2).

However, the main problem is detecting micrometastasis (cN0 pN1), which are found in up to $50 \%$ of patients with cN0 tongue squamous cell lesions who undergo neck dissection. For this reason, the treatment of N0 neck in early tongue squamous cell tumors is debated (3). Because of the morbidity associated with neck dissection (such as hemorrhage, nerve injury, pain, or lymphedema (4), several studies have compared a wait-and-see policy in patients with clinically N0 tumors with elective neck dissection, and the results showed a worse prognosis in cases that later develop $\mathrm{cN}+$ and undergo delayed neck dissection.
However, improved survival was observed in cN0 that was revealed to be pN1 after elective neck dissection $(5,6)$.

The real problem in these patients with cN0 disease is to know whether the nodes are truly negative or harbor occult metastases. That is why according to many researches the chance of occult or subclinical nodal metastases in cN0 early oral cancers can be up to $3 \%$ (4). Often such a patient is either undertreated or over treated for the neck. It would be ideal to do a neck dissection only in SLN positive patients and spare its morbidity in the rest (7).

Most SLN studies in oral cancers use lymphoscintigraphy with radiolabeled particles and gamma probe localization combined with a blue dye injection (8). However, lymphoscintigraphy and nuclear medicine facilities are not widely available in Egypt, therefore, alternate techniques for SLN Biopsy need evaluation. Methylene blue is a low cost, less allergic and effective dye that has shown promising results in SLN studies in breast cancer when used alone or in combination with lymphoscintigraphy (9). 
Metastatic cervical lymph nodes from head and neck carcinomas are usually site specific concerning the location of the primary tumor. Therefore, assessment of the distribution of metastatic nodes in patients with unknown primary may provide a clue to the site of the primary tumor. Moreover, metastatic nodes in an unexpected site indicate that the primary tumor is biologically more aggressive (10). The role of ultrasound in the assessment of cervical lymphadenopathy is well established. It is particularly sensitive and specific compared to clinical examination (96.8\% and $73.3 \%$ respectively) in patients with previous head and neck cancer (11).

This study expects high-accuracy rates for intraoperative frozen sections, but the reliability of frozen sections in predicting the ultimate final margin status is unknown $(11,12)$. Therefore, frozen and permanent reads will be compared to identify risk factors for overall discrepancies between intraoperative and final margin status. Diagnostic accuracy of frozen section histopathological analysis will be also tested in determining the free margins of excised tumors.

The objective of the prospective study was to evaluate the effectiveness of SLN using MBD alone and frozen section of early oral cancer with N0 neck patients in the identification of occult neck micrometastasis and confirmation of the role of SLN in post-surgical follow up. We proposed that SLN using MBD alone and frozen section of early oral cancer with N0 neck patients can be used to identify occult neck micrometastasis.

\section{MATERIALS AND METHOD}

A prospective study of SLN Biopsy with methylene blue dye injection was conducted on 12 patients with early oral squamous cell carcinoma (T1-T2) \{tumor size $\leq 4 \mathrm{~cm}$ \} and N0 neck as revealed by both clinical examination and preoperative ultrasonic were treated in the Maxillofacial and plastic surgery, Department, Faculty of Dentistry, Alexandria University. Institute ethics committee approval was obtained and informed consent taken from all participants after a detailed explanation of the study objectives, benefits, risks, confidentiality of data and right to withdraw any time with no penalties. The evaluation included history, clinical examination, ultrasound and CT scans of the neck, chest X-ray and blood analysis as appropriate. The patients underwent surgery after pre-anesthetic evaluation; exclusion criteria included oral cancer patients with T3\&T4 cancers \{tumor size $>4 \mathrm{~cm}$ with invasion\}, clinical node positivity, lesions in or crossing midline, patients with previous surgery/scar in the neck, patients with previous history of neck irradiation and medically compromised patients..

Under general anesthesia, methylene blue dye was injected peritumoral ( $0.5 \mathrm{ml}$ each in 3, 6, 9 \&12 O’clock positions) as a concentration of $0.1 \mathrm{gm}$ of methylene blue in $100 \mathrm{ml}$ of distilled water (Figure 1). Surgery for the neck was done first; a modified curvilinear neck incision was made from mentum to mastoid curved along the upper border of hyoid and midpoint of the sternocleidomastoid followed by flap elevation using electrocautery. Systematic evaluation of levels IA, IB, IIA, IIB, III \& IV nodes was done. Sentinel node (s) were identified by the blue dye staining within 20 minutes (Figure 2). SLN were dissected and sent for frozen section (FS). If SLN was negative on Frozen Section (FS) the patient underwent selective neck dissection (SND). If SLN was positive on Frozen section (FS), the patient underwent Modified Radical Neck Dissection (MRND) by converting the incision to a modified Crile's incision by the addition of a vertical limb. Surgery for the primary was then completed followed by immediate reconstruction. The tumor sent for frozen section evaluation of safety margins. Closure of the wounds in layers. All nodes in Levels I, IIa, IIb, III, IV (and V in MRND) including SLN (blue dye stained nodes) were dissected, marked separately and sent for histopathological examination (HPE) with serial step sectioning of paraffin fixed specimens and H\&E. IHC for pancytokeratin was performed for lymph nodes showing negative micrometastasis.

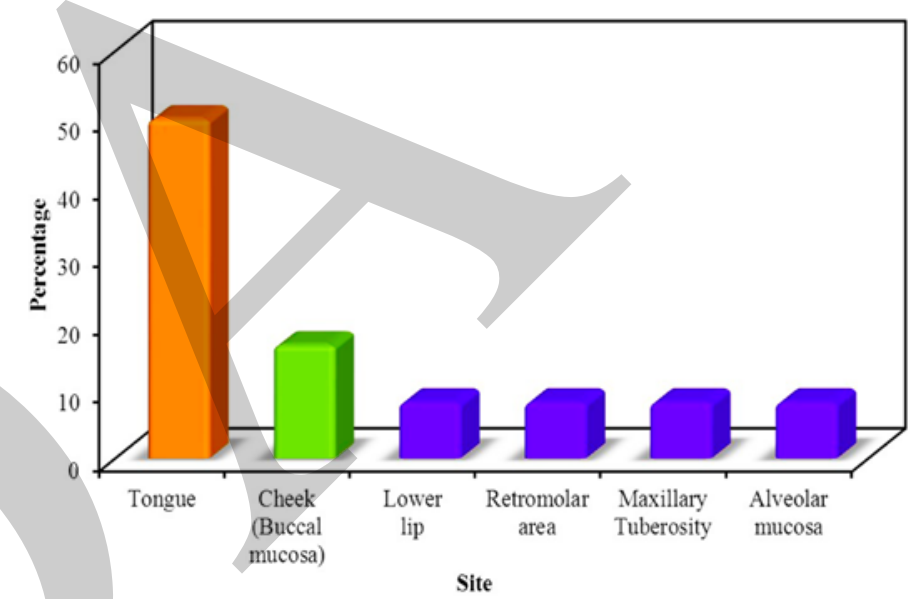

Figure 1: Injection of methelyn blue.

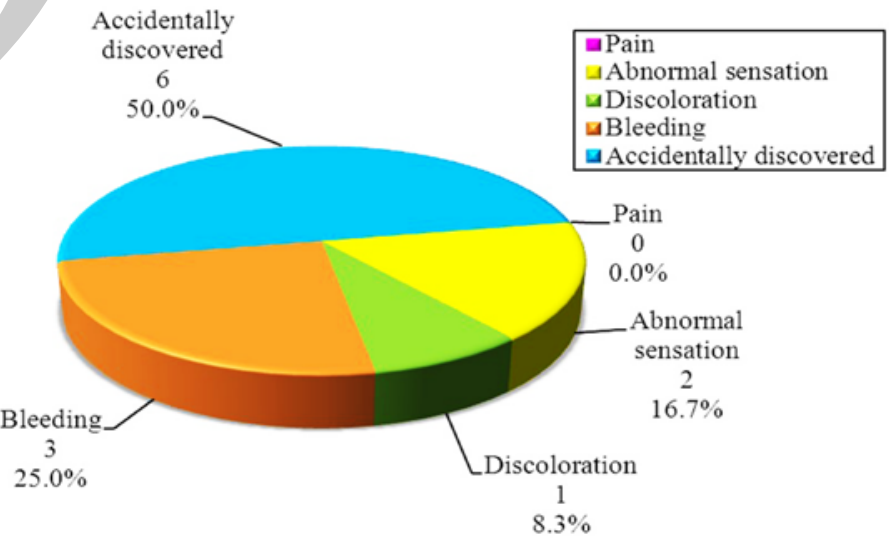

Figure 2: Intraoperative identification of SLN.

The patients were discharged to the ward for completing the postoperative care and treatment. The patients were clinically and radiographically followed up at one month, 3 months, 6 months, 12 months, and 18 months postoperative. During each visit, the patient was evaluated for the outcome of the operation 
using ultrasonography, signs of residuals, the occurrence of complications or any dehiscence (10).

\section{Statistical analysis}

Data were collected and entered to the computer using SPSS 16 (Statistical Package for Social Science) program for statistical analysis (IBM Inc., USA) and EXCEL 2007 (Microsoft, USA).

\section{RESULTS}

Twelve patients with early oral cancer were treated in the Maxillofacial and plastic surgery Department, Faculty of Dentistry, Alexandria University.

\section{Preoperative evaluation}

\section{$>\quad$ Age and sex distribution}

The age of the patients ranged from 38 years to 72 years with a mean of $55.42 \pm 13.50$. Eight patients were females (66.7\%), four (33.3\%) were males.

\section{$>$ Clinical presentation}

Twelve patients were presented with small oral ulcers. The tongue cases were $50 \%$ (6 cases) and the cheek mucosa was $16.7 \%$ ( 2 cases) with only one case lower lip, retro molar area, maxillary tuberosity and alveolar mucosa each is $8.3 \%$ (Figure 3). Most of the cases discovered accidentally during dental examination (6 cases) 50\% followed by a complaint of bleeding (3 cases) 25\% then (2 cases) with abnormal sensation 16.7\% and only ( 1 case) presented with discoloration $8.3 \%$ and none presented with pain (Figure 4).

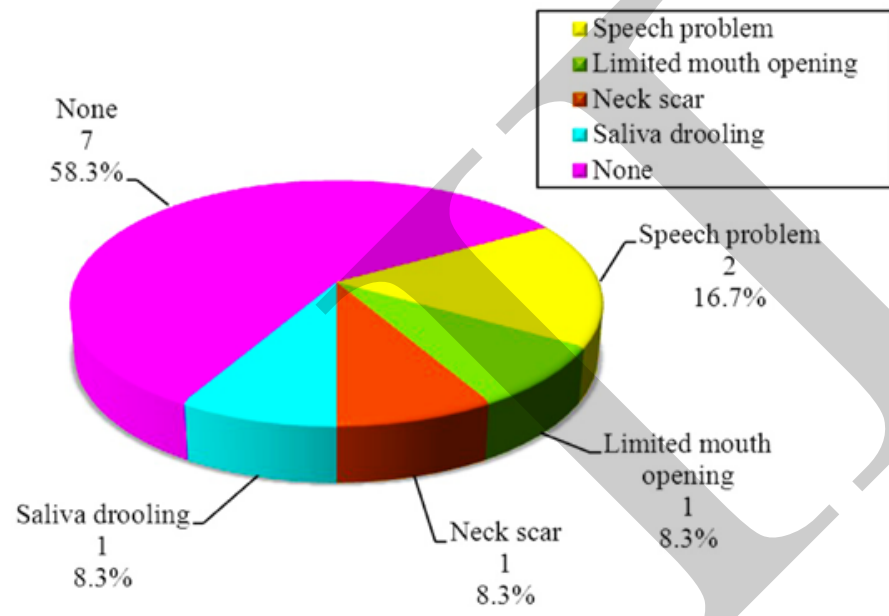

Figure 3: Distribution of the studied cases according to site $(n=12)$.

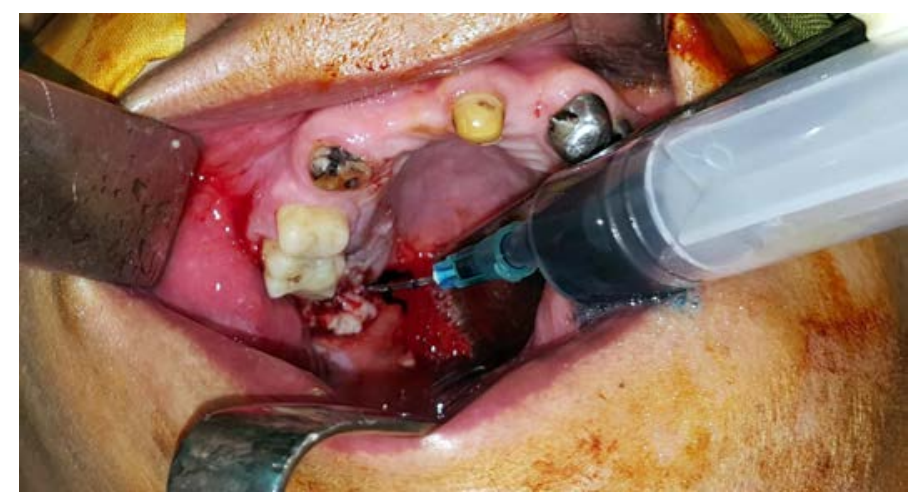

Figure 4: Distribution of the studied cases according to clinical presentation $(n=12)$.

\section{$>$ Intraoperative findings}

Three cases (25\%) sentinel lymph node couldn't be detected (Case 6, 8, 12), in which methylene blue did not stain any lymphoid tissue in the neck, while nine cases (75\%) the blue sentinel lymph node could be identified (Table 1). Preoperative ultrasound examination of only two cases were not similar to the intraoperative frozen histopathology which reveals the presence of micrometastasis, therefore functional neck dissection was indicated in these two cases. From other points, all the frozen sections histopathology were similar to the postoperative paraffin sections (Table 2).

Table 1: Distribution of the studied cases according to detection of SLN

\begin{tabular}{|c|c|}
\hline Case No. & Detection of SLN \\
\hline 1 & $\sqrt{ }$ \\
\hline 2 & $\sqrt{ }$ \\
\hline 3 & $\checkmark$ \\
\hline 4 & $\checkmark$ \\
\hline 5 & $\times$ \\
\hline 6 & $\checkmark$ \\
\hline 7 & $\times$ \\
\hline 9 & $\checkmark$ \\
\hline 10 & $\checkmark$ \\
\hline 11 & $\checkmark$ \\
\hline 12 & $\times$ \\
\hline
\end{tabular}

Table 2: Distribution of the studied cases according to detection of malignant micro-metastasis.

\begin{tabular}{||c|c|c||}
\hline Case No. & $\begin{array}{c}\text { Intraoperative frozen } \\
\text { histopathology }\end{array}$ & $\begin{array}{c}\text { Postoperative paraffin } \\
\text { histopathology }\end{array}$ \\
\hline $\mathbf{1}$ & Negative & Negative \\
\hline $\mathbf{2}$ & Negative & Negative \\
\hline $\mathbf{3}$ & Negative & Negative \\
\hline $\mathbf{4}$ & Positive & Positive \\
\hline
\end{tabular}




\begin{tabular}{||l|l|l||}
\cline { 2 - 3 } & Negative & Negative \\
\hline $\mathbf{6}$ & Negative & Negative \\
\hline $\mathbf{7}$ & Negative & Negative \\
\hline $\mathbf{8}$ & Negative & Negative \\
\hline $\mathbf{9}$ & Positive & Positive \\
\hline $\mathbf{1 0}$ & Negative & Negative \\
\hline $\mathbf{1 1}$ & Negative & Negative \\
\hline $\mathbf{1 2}$ & Negative & Negative \\
\hline
\end{tabular}

\section{Postoperative complications and follow up}

Long term follow up show only two cases suffered from speech problem for 3 months that was improved eventually later, One case suffered from limited mouth opening due to adjuvant radiotherapy that induced cheek fibrosis, Another case showed drooling of saliva postoperatively after lower lip excision that was significantly controlled by two years follow up, One female patient complained of neck scar that was improved by topical steroids and silicone gel. The rest of the cases follow up was unremarkable for complications (Figure 5)

The time taken for the lymphatic vessels and nodes to turn blue was recorded, it was $25 \pm 6$ minutes also it was noted that its excretion through kidney was in $50 \pm 8$ minutes was uneventful. No blue bye stained nodes (SLN) were identified in Level IIB and IV. None of the SLN nodes in Level IIB and IV had metastases on HPE and IHC. There were no skip metastases, extranodal disease or overflow phenomenon. Considering HPE on paraffin fixed blocks as the gold standard, FS had a sensitivity, specificity, and NPV of $95.8 \%$. IHC with cytokeratin increased the sensitivity (100\%) and NPV (100\%) of histopathology at the loss of specificity (87.5 \%) and PPV (62.5\%).

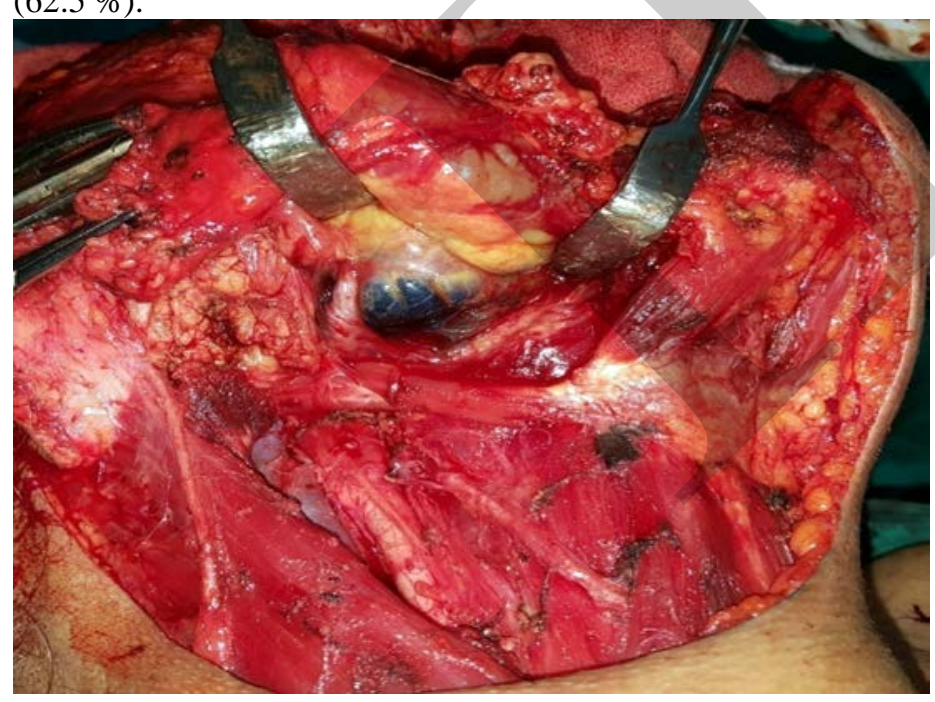

Figure 5: Distribution of the studied cases according to postoperative satisfaction for follow up.

The study shows the relationship between the results of intraoperative lymph node biopsy using the frozen section method and the actual existence of neck metastasis determined by postoperative histologic examination of patients undergoing neck dissection or by the clinical course of those not undergoing neck dissections. Intraoperative biopsy resulted in a correct diagnosis for all patients, whereas 2 were diagnosed as cN0 but had neck metastasis. Overall accuracy was therefore $96.7 \%$ and the false-negative rate was $3.3 \%$.

A limitation of our study is the lack of accurate anatomical information on the precise localization of lymph nodes on the baseline and follow-up ultrasound. This limited the observer in comparing each node. Therefore, the largest lymph node per level was used for comparison and calculation of the change in the size of the lymph nodes.

\section{DISCUSSION}

The theory behind SLN biopsy approach is that lymph flows from a tumor sequentially to the "first echelon node" (SLN) then to the remaining nodal basin, then, to next higher group of nodes, labeled in our study as station II nodes, from where lymph goes to next higher station nodes in an orderly way. SLNB is a technically demanding procedure, requires expertise and facilities like lymphoscintigraphy, gamma camera, etc. The advantages of SLNB include less morbidity and better cosmetic outcome with 2-year survival rates. Most SLN studies in oral cancers use lymphoscintigraphy with radiolabeled particles with gamma probe localization and blue dye injection (13).

Preoperative radionuclide scanning is associated with the risk of radioactive contamination. SLN identification in breast cancer using blue dye alone has been widely reported with identification rates slightly inferior or equal to combined techniques (14). Methylene blue is an effective and safer alternative to isosulphan blue dye in breast cancer (15). There are very few studies that assess the feasibility of using blue dye alone in detecting SLN in oral cancers. We used methylene blue dye for the study because of its lower cost, easier availability, good safety profile, and comparable efficacy. The time is taken to identify SLN also varied between $25 \pm 6$ minutes from dye injection. We especially experienced resistance in dye injection in the hard palate, retro molar area and alveolar lesions due to tough adherent mucoperiosteum.

There has been considerable debate regarding the method of pathological evaluation of SLN. Imprint Cytology, H\&E staining in paraffin-embedded blocks, Serial step sectioning of the paraffin blocks and Immunohistochemistry have been compared in their ability to detect occult metastases. Imprint Cytology is usually inferior to serial step section in the frozen section and IHC studies (16). Frozen section was used in our study to evaluate metastases in SLN and compared it with HPE based on serial step sectioning and IHC for cytokeratin. The sensitivity, specificity, and NPV of $95.8 \%$ of FS with methylene blue alone are also comparable to SLN studies using both radio colloid and blue dye.

The regional recurrence rate of the untreated neck of classification N0 is $20 \%$ to $30 \%$ among patients with early oral tongue carcinoma, and $90 \%$ of them occur within 20 months of the initial surgery (17). Treatment of clinically T1/T2 or N0 oral 
tongue carcinoma is controversial because the predominant opinion is that patients should undergo neck dissection if there is a risk of occult metastasis. Until now, elective neck dissection or a watchful waiting policy has controversy.

Elective neck dissection generally reduces mortality due to regional recurrence and also increases overall survival. If the watchful waiting policy is applied as the means of managing regional recurrence, clinicians must have the appropriate followup skills and be able to find smaller metastases that do not affect patients' prognosis. However, salvage surgery is unsatisfactory because it is successful in only $24 \%$ of patients (18). The validity of SNB for T1 or T2, clinically N0, oral cancer was tested in the American College of Surgeons Oncology Group (ACOSOG) trial involving 25 institutions in North America. Accuracy of SLNB was tested against neck dissection, which was performed at the same time in all patients. Results confirmed the conclusions of the European multicenter trial, as well as multiple single-center studies, that SNB is a reliable and reproducible means of staging the clinically NO neck in oral cancer (19). In our study, the "gold standard" for assessing the accuracy of SNB is recurrence noted on clinical follow-up. All patients have had the opportunity to be followed for a minimum of 2 years with a median follow-up of 18 months. NPV of 95.8 $\%$ is comparable to that observed in the ACOSOG and European trials. Ultrasonography scans applied by experienced clinicians are very helpful to evaluate nodal staging and are accurate diagnostic tools. All devices have advantages and disadvantages concerning detection abilities. Metastatic lymph nodes that are $<10 \mathrm{~mm}$ thick can be easily detected by ultrasonography, but are often overlooked by CT and MRI. However, ultrasonography cannot detect micro metastatic foci in lymph nodes or evaluate positive/negative lymph nodes if the shapes of lymph nodes do not transform during metastasis (20).

SND has achieved wide acceptance as a staging procedure, and for elective treatment of the neck. Its role has been expanded in some institutions to include treatment of patients with clinically evident lymph node metastases that is to say in the therapeutic treatment of neck (21). Although there is a paucity of outcomes data to indicate the efficacy of SND based on the extent of nodal disease, taking care that clinical nodal disease should be limited (N1 and selected N2 disease) and confined to the first and possibly second echelons of nodal drainage. Thus for oral cavity carcinoma, SND is appropriate for the presence of nodal disease confined to level I and II only. Similarly, for oropharyngeal and laryngeal carcinomas, the nodal disease should be confined to levels II-IV. Even with these guidelines, the presence of multiple nodes in more than one level indicates a higher risk for involvement of neck levels beyond the boundaries of SND, and MRND might be more appropriate, combined with postoperative radiation or chemo radiation (22).

In a review of 106 node-positive patients (N1- N2c) undergoing a SND, six patients experienced disease recurrence in the side of the neck that had undergone SND, resulting in a regional control rate of $94.3 \%$. The authors concluded that these results support the use of SND in carefully selected patients with clinically negative nodal metastasis from head and neck squamous cell carcinoma. The technique of SND has also been expanded to include removal of one or more of the nonlymphatic structures routinely included in the MRND or RND (i.e. sternocleidomastoid muscle, internal jugular vein, spinal accessory nerve). The structures most frequently sacrificed are the submandibular salivary gland and the internal jugular vein (23). In patients with tumors of the oral cavity with clinically N1 neck disease involving levels I or II. These findings strongly suggest that these patients could be treated with a SOHND (extended or not to level IV) (24). Van Agthoven et al. (25) in a retrospective study of patients with clinical evidence of neck metastases demonstrated comparable regional control rates in patients undergoing SND followed by radiotherapy as a similar group treated with MRND and radiotherapy.

The use of postoperative radiotherapy should be considered in patients with pathologically positive lymph nodes, particularly in cases with adverse prognostic factors such as multiple metastatic lymph nodes or any node with extracapsular spread, which may occur before involved lymph nodes are clinically or radiologically apparent. Several studies have noted the applicability of SND in $\mathrm{N}+$ disease when combined with radiotherapy postoperatively (26).

The poor prognosis in patients with microscopically positive cervical nodes after failed irradiation was demonstrated as early as 1979 by Silver and Croft (27) who performed therapeutic RND after failed irradiation. There was not a single survivor among 13 patients with histologically positive nodes. This study demonstrates that while the prognosis is poor in such patients, more radical procedures offer no advantage over SND. With the expansion of indications for SND which will likely play a greater role in the future for patients whose bulky nodal disease has initially been treated with radiotherapy or chemo radiation. Sentinel node navigation surgery applied at the clinical stage could detect metastatic foci before lymph nodes undergo extracapsular spread and the patients' prognosis would be increased. Furthermore, sentinel node navigation surgery (SNNS) can reduce the incidence of adverse events resulting in functional and cosmetic morbidities associated with neck dissection (28). We recommend sentinel node navigation surgery (SNNS) in the management of clinically N0 in early tongue carcinoma. Our findings showed that, although the reduction in regional recurrence was not statistically significant after SNNS compared with watchful waiting, SNNS tended to reduce regional recurrence. The extracapsular spread of positive lymph nodes an important prognostic factor. Unnecessary neck dissection can be avoided using SNNS and treatment outcomes can catch up with those of a policy of prophylactic neck dissection.

Elective prophylactic neck dissection (levels I-III) has one more disadvantage in that skip metastases are sometimes missed. From this viewpoint, SNNS is likely to become a more individualized approach than uniform neck dissection. The clinical and pathological findings of the primary tumor showed that $\mathrm{T}$ classification, histological grade, the lymphatic and 
venous invasion did not affect lymph node metastasis, whereas muscle invasion was required. T classification might not be any more important as a prognostic factor than tumor depth for patients with early oral tongue carcinoma. Sowder et al (29) suggested elective neck dissection for those with tumor depth $>4$ $\mathrm{mm}$ and Eskander et al (30) considered that tumor thickness rather than $\mathrm{T}$ classification might be the best way to select patients who require elective neck dissection.

Considering that the regional recurrence rate of tumors that reach $<1 \mathrm{~mm}$ into the muscle layer is $33 \%$ the extent of muscle layer invasion is not the key issue; only muscle invasion parse is important as a prognostic factor (31). The recommended level of management for clinical N0 in early oral tongue carcinoma can depend on SNNS. Further studies of larger populations and technical improvements are required to resolve the controversy. Sometimes, the limitation of intraoperative frozen section examination may lead to individual micro metastases being missed. Therefore, if future studies can find better alternative approaches to identify SLN. SLNB will have a higher clinical value. It has been reported that SLNB with step sectioning and immunohistochemistry can improve the detection rate of micro metastases.

Unfortunately, this method is difficult to apply to intraoperative rapid diagnosis. These problems are prevalent in the clinical application of SLNB; therefore, a rapid, accurate intraoperative test for the presence of metastatic disease in SLN may support widespread acceptance of SLNB in cases of SCC of the head and neck. We would like to emphasize the limitations of using methylene blue dye alone for SLNB. While using lymphoscintigraphy, the size of the neck incision can be minimized over hot spots before extending it for formal neck dissections. However, the mandatory elevation of the neck flaps before SLN identification while using methylene blue alone probably negates the idea of less morbidity associated with SLNB.

The other drawbacks are the inability to evaluate the contralateral neck and the chance of missing some deeper nodes. It is feasible that the extent of neck dissection can be reduced for SLN-negative patients. Larger multi-institutional trials will help us to better understand and address the drawbacks of this technique for its widespread applicability in decision making especially in countries like Egypt where lymphoscintigraphy is not widely available.

\section{CONCLUSIONS}

Using SLN biopsy allows for reducing the risk of occult lymph node metastases in T1/T2 oral cancer patients from $40 \%$ to $8 \%$, in addition, reducing unnecessary functional and esthetic complications for N0 patients by reducing the incidence or extent of neck dissection. Frozen section biopsy followed by Immunohistochemistry increase the sensitivity and negative predictive value of SLN but its applicability in real-time decision making is limited. None of the differences between watchful waiting and SLNB reached statistical significance, but SLNB tended to reduce the rate of regional recurrence.
Therefore we recommend that SLNB be the management strategy for treating clinical N0 in early oral cancer. The benefits of SLNB in the treatment of early tongue carcinoma should be further evaluated. Methylene blue dye alone can be successfully used for SLN identification in early oral cancers with accuracy and sensitivity. This method will be of use especially in resource-limited countries and centers where nuclear medicine facilities are not widely available.

\section{CONFLICT OF INTEREST}

The authors declare that they have no conflicts of interest.

\section{ACKNOWLEDGMENT}

My gratitude to my professors, colleagues, friends, students and last but not least my family.

\section{REFERENCES}

1. Crombie AK, Farah C, Tripcony L, Dickie G, Batstone MD. Primary chemoradiotherapy for oral cavity squamous cell carcinoma. Oral Oncol. 2012; 48:1014-8.

2. Samant S. Sentinel node biopsy as an alternative to elective neck dissection for staging of early oral carcinoma. Head Neck. 2014; 36:241-6.

3. Silverman DA, El-Hajj M, Strome S, Esclamado RM. Prevalence of nodal metastases in the submuscular recess (level IIb) during selective neck dissection. Arch Otolaryngol - Head Neck Surg. 2003; 129:724-8.

4. Ambrosch P, Brinck U. Detection of nodal micrometastases in head and neek cancer by serial sectioning and immunostaining. Oncology. 1996; 10:1221-6.

5. Melkane AE, Mamelle G, Wycisk G, Temam S, Janot F, Casiraghi O, et al. Sentinel node biopsy in early oral squamous cell carcinomas: A 10-year experience. Laryngoscope. 2012; 122:1782-8.

6. Dias FL, Kligerman J, Matos De Sá G, Arcuri RA, Freitas EQ, Farias $\mathrm{T}$, et al. Elective neck dissection versus observation in stage I squamous cell carcinomas of the tongue and floor of the mouth. Otolaryngol - Head Neck Surg. 2001; 125:23-9.

7. Suresh, T. N., Harendra Kumar, M. L., Thomas, A. K., \& Azeem, M. (2013). Study of sentinel lymph node in oral Squamous cell carcinoma. Journal of Biomedical Science. 2013; 3:146-9.

8. Kurita H, Koike T, Narikawa JN, Sakai H, Nakatsuka A, Uehara S, et al. Clinical predictors for contralateral neck lymph node metastasis from unilateral squamous cell carcinoma in the oral cavity. Oral Oncol. 2004; 40:898-903.

9. Kovács AF, Landes CA, Hamscho N, Risse JH, Berner U, Menzel C. Sentinel node biopsy as staging tool in a multimodality treatment approach to cancer of the oral cavity and the oropharynx. Otolaryngol - Head Neck Surg. 2005; 132:570-6.

10. Stoeckli SJ, Pfaltz M, Ross GL, Steinert HC, MacDonald DG, Wittekind C, et al. The Second International 
Conference on Sentinel Node Biopsy in Mucosal Head and Neck Cancer. In: Annals of Surgical Oncology. 2005; 12:919-24.

11. Vorburger MS, Broglie MA, Soltermann A, Haerle SK, Haile SR, Huber GF, et al. Validity of frozen section in sentinel lymph node biopsy for the staging in oral and oropharyngeal squamous cell carcinoma. J Surg Oncol. 2012; 106:816-9.

12. Chone CT, Aniteli MB, Magalhães RS, Freitas LL, Altemani A, Ramos CD, et al. Impact of immunohistochemistry in sentinel lymph node biopsy in head and neck cancer. Eur Arch Oto-Rhino-Laryngology. 2013; 270:313-7.

13. Hwang JM, Fu KK, Phillips TL. Results and prognostic factors in the retreatment of locally recurrent nasopharyngeal carcinoma. Int J Radiat Oncol Biol Phys. 1998; 41:1099-111.

14. Lubin JH, Purdue M, Kelsey K, Zhang ZF, Winn D, Wei Q, et al. Total exposure and exposure rate effects for alcohol and smoking and risk of head and neck cancer: A pooled analysis of case-control studies. Am J Epidemiol. 2009; 170:937-47.

15. Guzzo M, Locati LD, Prott FJ, Gatta G, McGurk M, Licitra L. Major and minor salivary gland tumors. Critical Reviews in Oncology/Hematology. 2010; 74:134-48.

16. Saloura V, Langerman A, Rudra S, Chin R, Cohen EEW. Multidisciplinary Care of the Patient with Head and Neck Cancer. Surgical Oncology Clinics of North America. 2013; 22:179-215.

17. Janoray G, Pointreau Y, Garaud P, Chapet S, Alfonsi M, Sire C, et al. Long-term results of a multicenter randomized phase III trial of induction chemotherapy with cisplatin, 5-fluorouracil, \pm docetaxel for larynx preservation. J Natl Cancer Inst. 2016; 108:djv368.

18. Forastiere AA, Zhang Q, Weber RS, Maor MH, Goepfert H, Pajak TF, et al. Long-term results of RTOG 91-11: A comparison of three nonsurgical treatment strategies to preserve the larynx in patients with locally advanced larynx cancer. J Clin Oncol. 2013; 31:845-852.

19. Haughey BH, Hinni ML, Salassa JR, Hayden RE, Grant DG, Rich JT, et al. Transoral laser microsurgery as primary treatment for advanced-stage oropharyngeal cancer: A United States multicenter study. Head Neck. 2011; 33:1683-94.

20. Bossi P, Alfieri S. The Benefit of a Multidisciplinary Approach to the Patient Treated with (Chemo) Radiation for Head and Neck Cancer. Current Treatment Options in Oncology. 2016; 17:53.

21. Tsai WC, Kung PT, Wang ST, Huang KH, Liu SA. Beneficial impact of multidisciplinary team management on the survival in different stages of oral cavity cancer patients: Results of a nationwide cohort study in Taiwan. Oral Oncol. 2015; 51:105-11.

22. Bergamini C, Locati L, Bossi P, Granata R, Alfieri S, Resteghini C, et al. Does a multidisciplinary team approach in a tertiary referral centre impact on the initial management of head and neck cancer? Oral Oncol. 2016; 54:54-7.

23. Alfieri S, Orlandi E, Bossi P. The Case Volume Issue in Head and Neck Oncology. Current Treatment Options in Oncology. 2017; 18:65.

24. Gatta G, Capocaccia R, Botta L, Mallone S, De Angelis R, Ardanaz E, et al. Burden and centralised treatment in Europe of rare tumours: results of RARECAREnet-a population-based study. Lancet Oncol. 2017; 18:1022-39.

25. Van Agthoven M, Van Ineveld BM, De Boer MF, Leemans CR, Knegt PP, Snow GB, et al. The costs of head and neck oncology: Primary tumours, recurrent tumours and longterm follow-up. Eur J Cancer. 2001; 37:2204-11.

26. Morselli P, Zollino I, Pinto V, Brunelli G, Carinci F. Clinical prognostic factors in stage I head and neck squamous cell carcinoma. J Craniofac Surg. 2008; 19:740-3.

27. Silver CE, Croft CB. Elective dissection of the neck. Surg Gynecol Obstet. 1979; 149:65-8.

28. Gourin CG, Johnson JT. A contemporary review of indications for primary surgical care of patients with squamous cell carcinoma of the head and neck. Laryngoscope. 2009; 119:2124-34.

29. Sowder JC, Cannon RB, Buchmann LO, Hunt JP, Hitchcock Y, Lloyd S, et al. Treatment-related determinants of survival in early-stage (T1-2N0M0) oral cavity cancer: A population-based study. Head Neck. 2017; 39:876-80.

30. Eskander A, Monteiro E, Irish J, Gullane P, Gilbert R, De Almeida J, et al. Adherence to guideline-recommended process measures for squamous cell carcinoma of the head and neck in Ontario: Impact of surgeon and hospital volume. Head Neck. 2016; 38:1987-92.

31. Tsai CJ, Zheng J, Zhang Z, Riaz N, Baxi SS, Wong RJ, et al. Association of number of dissected lymph nodes with survival in clinically node-negative oral cavity squamous cell carcinoma patients undergoing primary surgery: A population-based analysis. JAMA Otolaryngol - Head Neck Surg. 2017; 143:1049-52. 